\title{
La malattia di Fabry oggi
}

Giornale di Tecniche Nefrologiche e Dialitiche 2018, Vol. 30(I) 56-59

(C) The Author(s) 2018

Reprints and permissions:

sagepub.co.uk/journalsPermissions.nav

DOI: 10.1 I77/0394936218760839

journals.sagepub.com/home/gtn

\section{Renzo Mignani}

(S)AGE

La Malattia di Fabry e AIAF Onlus

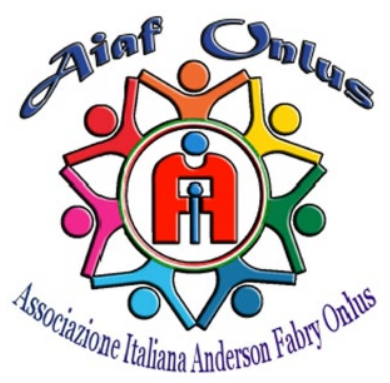

\begin{abstract}
Fabry disease is a multisystemic, X-linked recessive lysosomal storage disease caused by decreased activity of alphagalactosidase $A$ that results in lysosomal accumulations of the glycosphingolipid GL-3. Progressive glycolipid accumulation is initially reversible but leads with time to tissue damage and later to organ failure in the third to the fourth decade and early death. It is an underdiagnosed condition leading to a delayed start of therapies and consequently to a lack of efficacy.

Several aspects of the disease have been clarified by research but there still remains much to learn about specific issues. Practitioners should highly suspect Fabry disease in individuals presenting with the signs and symptoms mentioned above, along with the information gleaned from a thorough personal and family history and physical examination. Great effort has been made by screening studies, which should be extended to all the risk categories. The pathogenetic role of new mutations should be investigated. Preliminary studies have shown enzyme replacement therapy and chaperone therapy to be effective when started early. However, new therapies are coming on the horizon and this bodes well for the future of patients suffering from Fabry disease.
\end{abstract}

\section{Keywords}

Fabry disease, hereditary disease, lysosomial disease, genotype

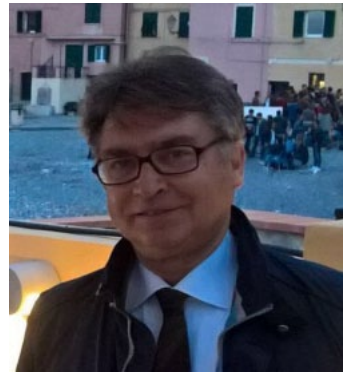

Nell'ambito della malattie rare di interesse nefrologico, la malattia di Fabry è quella che negli ultimi anni ha suscitato il maggiore interesse e divulgazione scientifica. Tutto ciò lo si deve indubbiamente all'avvento da oltre quindici anni della terapia enzimatica sostitutiva con agalsidasi. Tale terapia ha determinato un reale cambiamento della storia naturale della malattia, al punto che è sempre più concreta la possibilità di prevenire il danno d'organo responsabile della severa morbilità e mortalità, consentendo così un notevole miglioramento della sopravvivenza del paziente affetto.

Come noto, la malattia di Fabry è una malattia genetica legata al cromosoma X dovuta alla carenza dell'enzima lisosomiale $\alpha$-galattosidasi A. Questo enzima è deputato

UO Nefrologia e Dialisi, Ospedale Infermi, Rimini, Italia

\section{Corrispondenza:}

Dott. Renzo Mignani, UO Nefrologia e Dialisi, Via Settembrini 2,

Ospedale Infermi, Rimini, Italia.

E-mail: renzo.mignani@auslromagna.it 
alla degradazione degli spingolipidi che pertanto si accumulano in vari tessuti e organi determinando nel tempo un danno cellulare irreversibile che porta alla insufficienza funzionale di alcuni organi vitali per l'organismo come il cuore, il rene, il sistema nervoso centrale.

In tutti questi anni gran parte della ricerca scientifica non si è concentrata solo sullo studio dell'efficacia delle varie terapie al momento disponibili ma si è anche indirizzata e ha conseguito notevoli risultati su ogni aspetto della malattia. Questo approccio ha consentito un notevole miglioramento delle conoscenze sulla diagnosi, sugli aspetti genetici e patogenetici, oltre che sulla modalità di monitoraggio del paziente.

In particolare, lo sviluppo e l'estensione degli screening sia nei neonati che negli individui a rischio (pazienti con insufficienza renale cronica, con ictus giovanili, con cardiomiopatia ipertrofica) ha permesso di scoprire nuovi casi, aggiornando così i vecchi dati sulla prevalenza della malattia. Lo sviluppo della tecnica del DBS (Dry Blood Spot) o della "goccia secca" ha velocizzato e reso ancor più facile lo svolgimento di tali studi di screening. Ma a favorire la diagnosi è stata anche la notevole opera di divulgazione della malattia promossa in questi anni: i numerosi eventi scientifici riguardanti i vari aspetti della malattia e svolti a livello locale, nazionale o internazionale hanno consentito una maggior conoscenza e consapevolezza della malattia soprattutto nella classe medica, permettendo di conseguenza una diagnosi sempre più precisa e precoce. In tal modo l'intervallo tra l'epoca di comparsa dei sintomi e la diagnosi della malattia si è sensibilmente ridotto, anche se tuttora non sempre la diagnosi viene fatta in età pediatrica. Sotto questo aspetto quindi c'è ancora molto da lavorare perché la diagnosi, e il conseguente avvio della terapia, sia sempre più tempestiva e precoce.

Dal punto di vista genetico, al momento sono oltre 700 le mutazioni individuate come responsabili della malattia. Non tutte però hanno dimostrato la possibilità di indurre uno stato di malattia. Infatti, uno degli aspetti più attuali e controversi è senza dubbio la discriminazione tra mutazioni cosiddette patogene, perché responsabili della malattia, e mutazioni non-patogene o causative. Tra queste ultime si annoverano i polimorfismi, le pseudo-deficienze in cui anche una eventuale riduzione dell'attività enzimatica non è associata all'accumulo di substrato sfingolipidico nei lisosomi cellulari. Tuttavia, la presenza in molti di questi polimorfismi di sintomi o segni clinici tipici della malattia rende spesso difficile l'interpretazione e l'attribuzione alla malattia di tale sintomatologia e il conseguente avvio della terapia. Pertanto serve molta attenzione, soprattutto in caso di nuove mutazioni, nell'attribuire la patogenicità di una nuova variante genica che dovrebbe prima ancora ad essere investigata a fondo, soprattutto con indagini istologiche mirate.

Gli studi sulla patogenesi della malattia hanno consentito di appurare come il semplice accumulo di substrato non sia da solo sufficiente a determinare il danno cellulare. Il coinvolgimento del sistema immunitario e aspetti come la flogosi, attraverso il rilascio di fattori pro-infiammatori, e l'autofagia sono stato indagati; sembra che possano avere un ruolo altrettanto importante nell'indurre il danno cellulare responsabile nelle fasi finali del processo della sclerosi o della fibrosi che si pone alla base del deficit funzionale degli organi maggiori coinvolti nella malattia.

Sotto l'aspetto terapeutico, dopo l'introduzione dell'agalsidasi oltre 16 anni fa gli studi di efficacia della terapia enzimatica si sono moltiplicati e continuano ad essere prodotti con crescente intensità e rilevanza scientifica. Al momento, sia gli studi tratti dai dati dei registri internazionali sia gli studi multicentrici hanno confermato la possibilità che tale terapia possa prevenire, stabilizzare o rallentare comunque la progressione del danno istologico e funzionale degli organi coinvolti (soprattutto rene, cuore e sistema nervoso periferico). Alcuni di questi studi in particolare hanno dimostrato la capacità della terapia enzimatica di rimuovere pressoché totalmente l'accumulo di substrato negli elementi cellulari più importanti del danno d'organo (come i podociti e i cardiomiociti) e conseguentemente di normalizzare iniziali segni di danno d'organo come l'albuminuria - soprattutto se l'avvio della terapia avviene in età pediatrica o adolescenziale. Infatti, la malattia di Fabry è una malattia da accumulo in cui l'impatto del deposito di substrato sul danno istologico e funzionale dell'organo è limitato e totalmente reversibile nelle prime decadi di vita. Pertanto, se l'avvio della terapia si verifica in queste fasce d'età è possibile non solo rimuovere la maggior quantità di depositi lisosomiali ma addirittura è possibile regredire un eventuale danno cellulare e conseguentemente funzionale dell'organo. Al contrario, se l'avvio del trattamento avviene tardivamente in presenza cioè di un danno cellulare diventato oramai irreversibile per il persistente e progressivo accumulo del substrato, la terapia può solo rallentare la progressione del danno cellulare verso l'insufficienza funzionale totale dell'organo. Tale concetto, sempre più attuale, era stato ben espresso, forse per primo, dal professore Barry Brenner, uno dei padri e pionieri della nefrologia moderna mondiale, che già nel 2004, prima ancora che si conoscessero gli effetti a lungo termine della terapia enzimatica da poco introdotta, aveva ipotizzato come solo una diagnosi precoce e quindi un avvio precoce della terapia possa prevenire il danno tissutale e la funzione dell'organo coinvolto (figura 1).

Di recente è stata introdotta la nuova e tanto attesa terapia chaperonica, che attraverso la somministrazione orale di una sostanza di origine glucidica è in grado di rendere stabile e funzionalmente attiva una seppur modica quantità di enzima $\alpha$-galattosidasi $\mathrm{A}$ residua. Tale terapia rappresenta indubbiamente un notevole passo in avanti nell'armamentario terapeutico della malattia e un vantaggio nella compliance del paziente. Tuttavia, soltanto una piccola parte di pazienti ne può beneficiare (coloro cioè che 


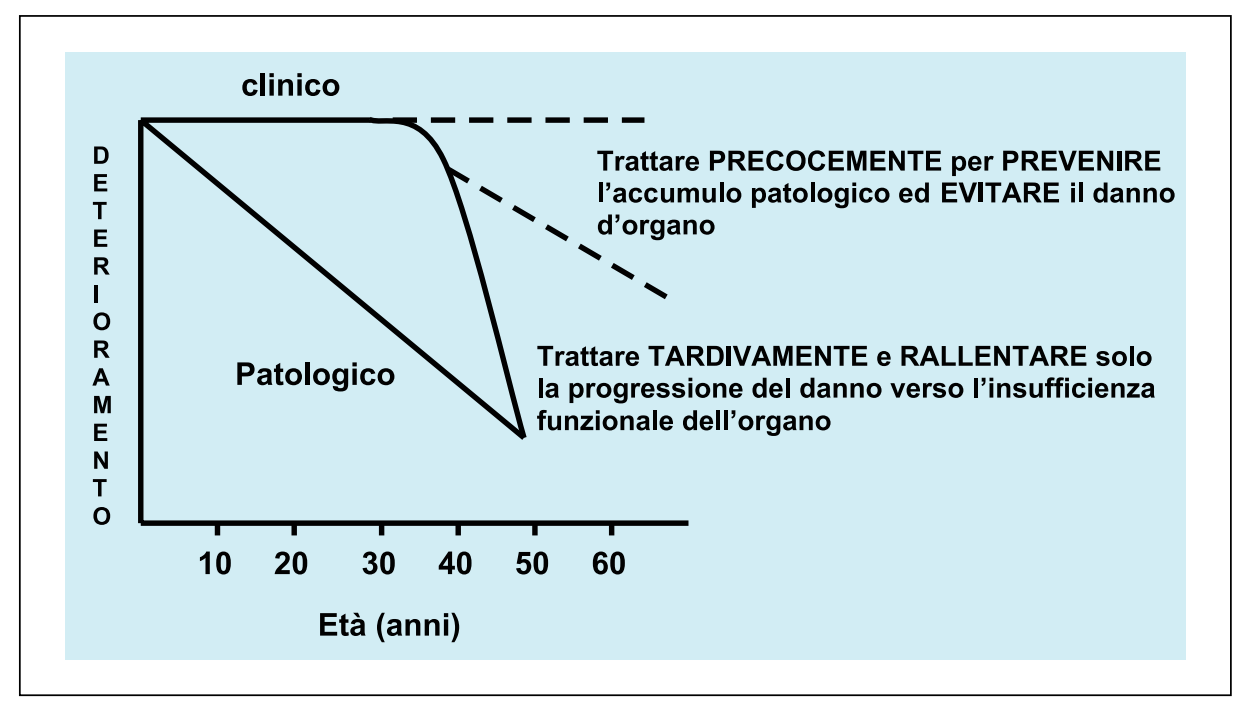

Figura I. Modificata da: Brenner B e Grünfeld J-P.'

sono affetti da mutazioni suscettibili e, di conseguenza, in grado di produrre ancora una seppur modica quantità di enzima $\alpha$-galattosidasi A funzionalmente non attivo) e al momento ne sono esclusi pazienti pediatrici. È bene quindi dare una corretta informazione al paziente in merito per non creare false aspettative. Oltre alla terapia chaperonica, sono attualmente in studio altri farmaci, altre terapie enzimatiche sostitutive di origine vegetale, farmaci in grado di ridurre il substrato con un meccanismo diverso dalla terapia enzimatica, che nei prossimi anni vedranno la luce ampliando così il bouquet di terapia disponibile e consentendo magari una vera e propria "personalizzazione" della terapia. Ma il futuro non troppo lontano sarà quello della terapia genica in grado di modificare (in utero?) e normalizzare il gene responsabile della malattia, ripristinando così una normale produzione enzimatica. Di tale trattamento innovativo è attualmente in corso una sperimentazione oltreoceano ma ancora le informazioni al riguardo sono scarse e inconclusive.

Ma altri passi in avanti sono stati fatti nel monitoraggio clinico del paziente che sia o no in trattamento con la terapia enzimatica o chaperonica. Come detto, la stabilità clinica del paziente rappresenta un importante obiettivo della terapia, soprattutto quando non è possibile ottenere la normalizzazione di certi parametri. La complessità della malattia per la sua natura multi sistemica fa sì che non sia sempre facile stabilire nella periodicità dei controlli clinici se un paziente sia stabile o non stabile, se la malattia tenda a manifestarsi in un paziente asintomatico o se la terapia sia efficace o no. Lo sviluppo di indicatori o di modelli matematici ha "modernizzato" la gestione clinica del paziente aiutando cosi il clinico che comunque rimane l'unico e il solo in grado di dare un giudizio clinico definitivo ponderato ed equilibrato sullo stato del paziente.
Infine, non si può non menzionare il ruolo che i pazienti stessi e la relativa associazione stanno svolgendo sullo sviluppo della conoscenza della malattia. La costituzione, finalmente, di una unica associazione nazionale pazienti AIAF e la intensa attività associativa, relazionale, comunicativa oltreché scientifica che in questi ultimi anni è stata svolta ha consentito ad AIAF di assumere un ruolo e un coinvolgimento sempre più frequente in varie attività promozionali e divulgative della comunità scientifica che si occupa della malattia.

In tutto ciò il contributo della comunità scientifica italiana è stato sempre molto forte ed incisivo sia nell'ambito della ricerca scientifica sia nell'attività di informazione $\mathrm{e}$ divulgazione contribuendo notevolmente al miglioramento delle conoscenze sulla malattia. Ed è proprio sulla base dell'interesse sempre più crescente suscitato da tale affascinante malattia, che ricordiamo coinvolge medici di tante specializzazioni, che il gruppo editoriale del Giornale di Tecniche Nefrologiche e Dialitiche ha pensato di inserire all'interno della rivista una rubrica periodica anche per la malattia di Fabry. È stato quindi costituito un comitato editoriale, che mi onora coordinare, cui fanno parte alcuni degli esperti italiani di riconosciuta competenza e autorevolezza scientifica a livello nazionale ed internazionale, al quale è stato affiancata la presenza dell'attuale presidente dell'associazione pazienti AIAF.

In questa rubrica, rivolta a medici, infermieri, pazienti, famigliari ma anche a chiunque voglia avvicinarsi alla malattia, verranno quindi trattati non solo aspetti clinici inerenti ai vari organi coinvolti dalla malattia, ma anche e soprattutto aspetti comunicativi, narrativi dando ampio spazio all'attività dell'associazione pazienti che mediante interviste con pazienti o familiari potrà trasmettere a tutti le esperienze personali vissute a causa della malattia. 
In tal modo, cercheremo di diffondere ulteriormente la conoscenza della malattia con un linguaggio chiaro, semplice, diretto ma nel contempo anche preciso e corretto che possa così avvicinare sempre più persone, addetti o no, alla conoscenza della malattia di Fabry.

\section{Dichiarazione di assenza di conflitto di interessi}

L'Autore dichiara di non avere conflitti di interessi.

\section{Finanziamenti}

L'Autore dichiara di non aver ricevuto finanziamenti specifici da qualsiasi ente nei settori pubblico, privato o senza fini di lucro.

\section{Bibliografia}

1. Brenner B e Grünfeld J-P. Renoprotection by enzyme replacement therapy. Curr Opin Nephrol Hy 2004; 13: 240. 